\title{
Observation of a Dissipation-Induced Classical to Quantum Transition
}

\author{
J. Raftery, ${ }^{1, *}$ D. Sadri, ${ }^{1, \dagger}$ S. Schmidt, ${ }^{2}$ H. E. Türeci, ${ }^{1}$ and A. A. Houck ${ }^{1}$ \\ ${ }^{1}$ Department of Electrical Engineering, Princeton University, Princeton, New Jersey 08544, USA \\ ${ }^{2}$ Institute for Theoretical Physics, ETH Zurich, 8093 Zurich, Switzerland
}

(Received 10 December 2013; revised manuscript received 16 May 2014; published 8 September 2014)

\begin{abstract}
Here, we report the experimental observation of a dynamical quantum phase transition in a strongly interacting open photonic system. The system studied, comprising a Jaynes-Cummings dimer realized on a superconducting circuit platform, exhibits a dissipation-driven localization transition. Signatures of the transition in the homodyne signal and photon number reveal this transition to be from a regime of classical oscillations into a macroscopically self-trapped state manifesting revivals, a fundamentally quantum phenomenon. This experiment also demonstrates a small-scale realization of a new class of quantum simulator, whose well-controlled coherent and dissipative dynamics is suited to the study of quantum many-body phenomena out of equilibrium.
\end{abstract}

DOI: 10.1103/PhysRevX.4.031043

An understanding of the physics of systems far from equilibrium [1] encompasses deep issues of fundamental importance such as dissipation, decoherence, emergence of classicality from intrinsically quantum systems [2], symmetry breaking and bifurcations, and how equilibrium is itself established [3-6]. Unraveling this intricate physics is essential to making sense of the world around us, which is fundamentally nonequilibrium and yet displays complex emergent structure. Much of the important recent progress in experimental condensed matter physics has explored the equilibrium regime of strongly correlated synthetic matter (e.g., ultracold atoms in optical lattices [7]), but it has been a long-standing goal to understand what new phenomena may arise as these systems are pushed away from equilibrium. With the rapid technological advances in solidstate quantum optics [8,9], it is now becoming possible to experimentally study strongly correlated photons, and to build model systems whose open nature gives rise to rich emergent behavior. Interaction with an environment has been argued to provide a mechanism for the emergence of classical behavior [2] from a quantum system. It is also possible, as our work explicitly demonstrates, that dissipation into an environment can qualitatively change this picture, where initially classical dynamics crosses over into one which is fundamentally quantum in nature.

Linear Josephson oscillations [10-14] and their anharmonic generalizations when interparticle interactions are

\footnotetext{
*jraftery@alumni.princeton.edu

†sadri@alum.mit.edu
}

Published by the American Physical Society under the terms of the Creative Commons Attribution 3.0 License. Further distribution of this work must maintain attribution to the author(s) and the published article's title, journal citation, and DOI.
Subject Areas: Condensed Matter Physics, Photonics, Quantum Physics

relevant [15-17] have been observed for atomic BoseEinstein condensates (BECs) [18] and more recently in a system of exciton polaritons [19]. At high density in such BEC systems, the large interactions dominate the tunneling and lead to macroscopic quantum self-trapping [20,21].

In this experiment, we explore a localization transition in a dissipative photonic system [22] realized in the circuit QED architecture [8,9], a solid-state realization of cavity QED [23]. As a system supporting phase-coherent photonic states and controlled nonlinearity (tunable in situ on nanosecond time scales) reaching well into the strongcoupling regime even at the single-photon level, it opens up the possibility of experimental condensed matter physics with strongly correlated photons. The flexibility in engineering model Hamiltonians and environmental couplings makes it an exemplary candidate for carrying out certain classes of quantum simulations [24,25] of important but difficult to study problems [26-31]. The dynamics of polaritons in driven dissipative Jaynes-Cummings chains have been studied theoretically, where a transition from classical to nonclassical steady-state fields, with varying interaction, tunneling and drive strengths, observable in the density-density correlation functions, have been suggested [32-34].

The physics of a single qubit coupled to a superconducting microwave resonator is well described by the JaynesCummings Hamiltonian (we choose units where $2 \pi \hbar=1$ ),

$$
\hat{H}^{\mathrm{JC}}=\nu_{c} \hat{a}^{\dagger} \hat{a}+\nu_{a} \hat{\sigma}^{+} \hat{\sigma}^{-}+g\left(\hat{\sigma}^{+} \hat{a}+\hat{\sigma}^{-} \hat{a}^{\dagger}\right)
$$

with $\nu_{c}\left(\nu_{a}\right)$ the bare cavity (qubit) frequency, $g$ the qubitcavity coupling rate, $\hat{a}, \hat{a}^{\dagger}$ representing the photon annihilation and creation operators, and $\hat{\sigma}^{ \pm}$the Pauli pseudospin operators. The photon-qubit interaction induces 
an anharmonicity in the spectrum of the Jaynes-Cummings Hamiltonian that leads to an effective on-site repulsion for photons [30]. Multiple Jaynes-Cummings sites can be coupled to form a lattice with various symmetries and topologies [26,27,36-38]. Here, we study the smallest nontrivial chain, coupling a pair of identical JaynesCummings sites through a photon hopping term (with rate $J$, and subscript $s=L / R$ specifying the left and right sites) to form a dimer [22]:

$$
\hat{H}_{\text {dimer }}=\sum_{s=L / R} \hat{H}_{s}^{\mathrm{JC}}-J\left(\hat{a}_{L}^{\dagger} \hat{a}_{R}+\hat{a}_{R}^{\dagger} \hat{a}_{L}\right) .
$$

Interaction with the environment is described through a Markovian Lindblad master equation governing the dynamics of the reduced density matrix of the dimer,

$$
\frac{\partial \hat{\rho}}{\partial t}=i\left[\hat{\rho}, \hat{H}_{\text {dimer }}\right]+\sum_{i=L, R}\left(\frac{\kappa}{2} \mathcal{L}\left[\hat{a}_{i}\right]\right)+\left(\frac{\gamma}{2} \mathcal{L}\left[\hat{\sigma}_{i}^{-}\right]\right),
$$

where the Liouvillian superoperator $\mathcal{L}[\hat{O}]=2 \hat{O} \hat{\rho} \hat{O}^{\dagger}-$ $\hat{O}^{\dagger} \hat{O} \hat{\rho}-\hat{\rho} \hat{O}^{\dagger} \hat{O}$ describes the cavity photon and qubit relaxation rates at $\kappa$ and $\gamma$, respectively. Dephasing for our choice of qubits (transmons) can be made much weaker than the above two channels $[39,40]$, and hence is ignored in our theoretical description.

We first discuss the semiclassical dynamics of the dimer in the absence of dissipation $(\kappa=\gamma=0)$. This can be done via the Heisenberg equations of motion and fully factorizing expectation values of spin-photon operator products, yielding a set of eight coupled differential equations for expectation values of the qubit and cavity field operators. A useful representation is in terms of real and imaginary parts of the cavity field $R_{s}=\operatorname{Re}\left\langle\hat{a}_{s}\right\rangle, I_{s}=\operatorname{Im}\left\langle\hat{a}_{s}\right\rangle$; with the angles parametrizing the spin direction $\vec{n}$ on the qubit Bloch sphere, $\vec{n}_{s}=\left[\sin \left(\theta_{s}\right) \cos \left(\phi_{s}\right), \sin \left(\theta_{s}\right) \sin \left(\phi_{s}\right), \cos \left(\theta_{s}\right)\right]$, these equations are

$$
\begin{aligned}
\dot{R}_{s} & =-\frac{g}{2} \sin \left(\theta_{s}\right) \sin \left(\phi_{s}\right)-J I_{\bar{s}}, \\
\dot{I}_{s} & =-\frac{g}{2} \sin \left(\theta_{s}\right) \cos \left(\phi_{s}\right)+J R_{\bar{s}},
\end{aligned}
$$

for the dynamics of the cavity ( $\bar{s}$ denotes the cavity opposite to $s$ ), and

$$
\begin{aligned}
& \dot{\phi}_{s}=-2 g\left[R_{s} \cos \left(\phi_{s}\right)-I_{s} \sin \left(\phi_{s}\right)\right] \cot \left(\theta_{s}\right), \\
& \dot{\theta}_{s}=-2 g\left[R_{s} \sin \left(\phi_{s}\right)+I_{s} \cos \left(\phi_{s}\right)\right],
\end{aligned}
$$

for the qubits. In writing these equations, we assume the qubits to be resonant with the respective cavity modes they are coupled to $\left(\nu_{a}=\nu_{c}\right)$, and work in the rotating frame. We define the photon number on the left and right as $N_{L / R}=\left\langle\hat{a}_{L / R}^{\dagger} \hat{a}_{L / R}\right\rangle$, the total photon number as their sum $N=N_{L}+N_{R}$, and the photon imbalance as $Z=\left(N_{L}-N_{R}\right) / N$. For special choices of initial conditions, the dynamics can be restricted to certain submanifolds of the phase space. One possible choice, $I_{1}=$ $R_{2}=0$ and $\phi_{1}=\pi / 2, \phi_{2}=0$, leads to a set of four coupled equations, which preserve this choice. This submanifold contains the dynamics corresponding to an initial condition with perfect imbalance (e.g., $Z=1$ for $R_{1}=\sqrt{N}$ and $I_{2}=0$ at $t=0$ ).

In the absence of qubit-cavity interaction $(g=0)$, the reduced set of equations can be solved exactly, giving rise to harmonic coherent Josephson oscillations of the imbalance at frequency $\nu_{J}=2 J$. With increasing coupling $g$, the oscillations become anharmonic. Solving the system of differential equations numerically (subject to the initial condition with $Z=1$ ) shows that, at a classical critical coupling [41],

$$
g_{c}^{c l} \approx 2.8 J \sqrt{N},
$$

the oscillation period diverges, exhibiting critical slowingdown, and resulting in a sharp crossover between two qualitatively different regimes of classical dynamical behavior [22], signaling a dynamical phase transition. For couplings beyond the critical value, the system localizes, with the initial photons trapped nearly entirely on a single site, spontaneously breaking the left-right symmetry. As the parameters $g$ and $J$ are fixed for a particular device, it is helpful to recast the problem in terms of a corresponding classical critical photon number $N_{c}^{\mathrm{cl}} \approx 0.13(\mathrm{~g} / \mathrm{J})^{2}$ for a given $g / J$. In the classical analysis, a dimer initialized with a photon number $N<N_{c}^{\mathrm{cl}}$ is expected to remain in the localized regime (noting also that the numerical prefactor determining the critical photon number is itself somewhat sensitive to the initial state.)

We now discuss the full quantum dynamics of the dimer in the absence of dissipation $(\gamma=\kappa=0)$. High-quality microwave generators acting as classical coherent sources prepare coherent states having nonzero homodyne voltages, making it possible to monitor the system by observing the homodyne quadratures $\hat{I}=(1 / 2)\left(\hat{a}+\hat{a}^{\dagger}\right)$ and $\hat{Q}=(i / 2)\left(\hat{a}^{\dagger}-\hat{a}\right)$. [Throughout this paper, we define the homodyne signal as $\xi=\langle\hat{I}\rangle^{2}+\langle\hat{Q}\rangle^{2}$, whereas the photon number is arrived at by averaging after squaring the individual quadratures, i.e., $\left\langle\hat{I}^{2}+\hat{Q}^{2}\right\rangle$. Note that the variables appearing in the classical equations of motion (4) and (5) are the expectation values of the quadrature operators.] In the limit $g \rightarrow 0$ with finite $J$, initializing the system with a coherent state leads to oscillations of coherent states between the two cavities with a fixed phase difference of $\pi / 2$. The oscillations here closely match the expected classical behavior of two coupled oscillators. Keeping $g$ finite and taking $J \rightarrow 0$, the two JaynesCummings sites decouple, leading to the well-known resonant collapse and revival phenomenon for a coherent state interacting with a single qubit [23]. From the point of view of the cavity, collapse and revival is a manifestation of the formation of a Schrödinger cat state, as each component 
(a)

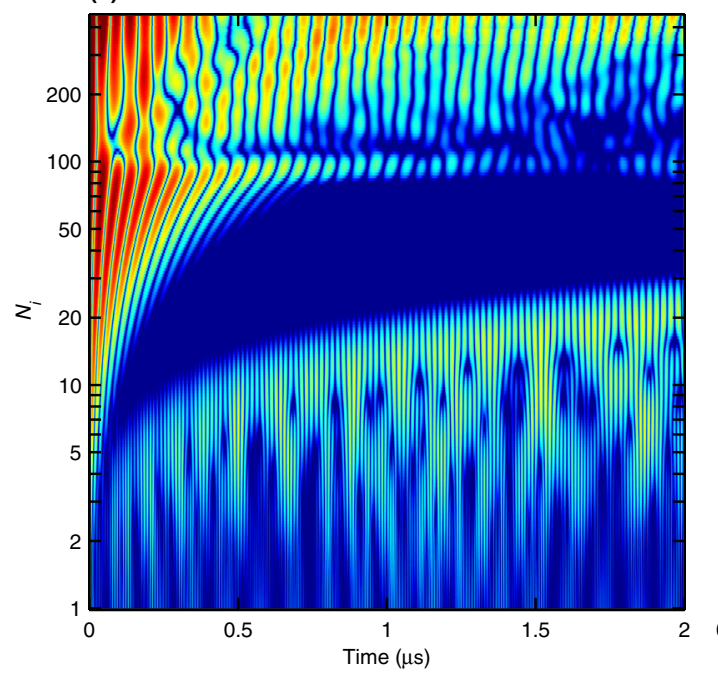

(b)

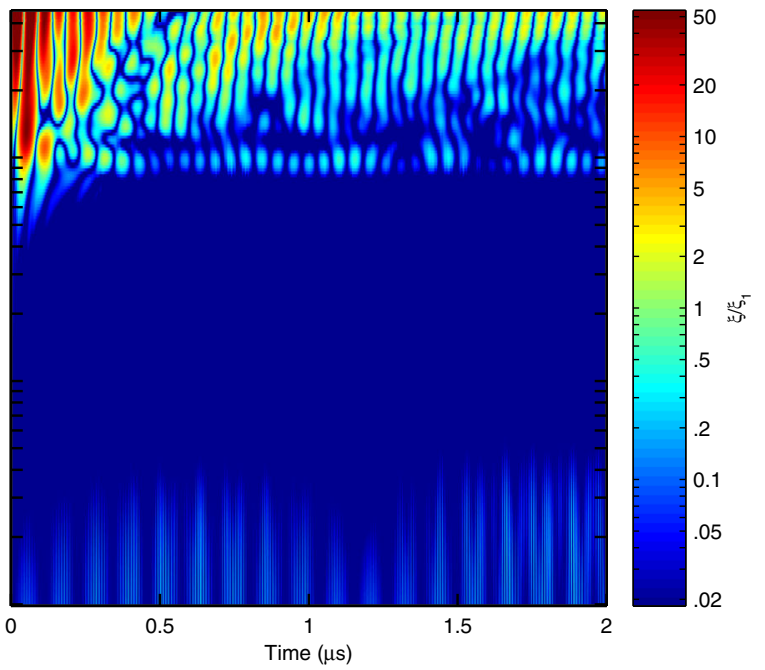

FIG. 1. Numerically simulated phase diagram. Quantum dynamics (without dissipation) of the homodyne signal in left (a) and right (b) cavities, as a function of total photon number (logarithmic vertical axis, with color bar normalized by $\xi_{1}$, the homodyne signal of a coherent state with mean occupation of one photon), for the case where the system has been initialized into the $Z=1$ imbalance state, photons in a perfect coherent state, and both qubits initially in the ground state. The parameters chosen are as given in the text corresponding to the actual device used in the experiment. For photon numbers below the quantum renormalized critical number $N_{c}^{\mathrm{qu}}$, tunneling is dynamically suppressed and we observe collapse-revival oscillations with period scaling as $t_{r}=\sqrt{N} / g$, as expected for decoupled single-site Jaynes-Cummings physics [23]. At very small photon numbers, we see an "exiguous" regime where tunneling reappears. We also observe in this localized regime secondary revivals at long times (also present in the unitary evolution of the single-site Jaynes-Cummings model), which would be washed out in the presence of dissipation. The dynamics above the critical photon number displays Josephson-like oscillations with period $t_{J}=(2 J)^{-1}$, becoming more linear with increasing photon number. We note that the critical number is marked by the coincidence of two time scales, $t_{r}=t_{J}$. As the initial $N$ increases, the revival period grows and ultimately matches that of the Josephson oscillation time scale, yielding the critical photon number $N_{c}^{\mathrm{qu}} \approx g^{2} / 4 J^{2} \approx 2 N_{c}^{\mathrm{cl}}$, consistent with where the transition is observed in the simulation. Solving the dynamics at the upper limit shown necessitates the solution of the time-dependent Schrödinger equation in a Hilbert space exceeding a dimension of size $10^{6}$. More details regarding the difficulty of such simulations can be found in the Supplemental Material [45].

of the cat state accumulates a different phase due to the interaction with the qubit $[43,44]$. The use of coherent states emphasizes the stark contrast between the two dynamical regimes-one characterized by classical oscillations and a second one characterized by the spontaneous formation of the quintessential macroscopic quantum mechanical state, the Schrödinger cat, displaying collapse and quantum revivals. These two regimes are demarcated by a dynamical quantum phase transition, with the localization a manifestation of macroscopic quantum selftrapping [16]. Here, we use the term dynamical quantum phase transition to describe a situation where a qualitative change occurs in the properties of the excited states as a function of a Hamiltonian parameter (here, $g / J$ ), instead of the ground state as in generic quantum phase transitions. The consequence of such a structural change in excited many-body states is reflected in the dynamics of appropriate observables after a quantum quench.

Inclusion of quantum fluctuations results in a renormalization of the critical coupling to its quantum value $g_{c}^{\text {qu }}$ (and likewise for the critical number to $N_{c}^{\mathrm{qu}}$ ). In Fig. 1, we show the numerically calculated quantum dynamics of the homodyne signal $\xi$ for initialization of the left cavity with a coherent state of the photon field of varying initial photon numbers (the qubits start out in the ground state and the right cavity in the vacuum state). We note that for the homodyne signal, while the delocalized regime is characterized by harmonic Josephson oscillations at frequency $\nu_{J}=2 J$ as for the imbalance $Z$, the localized regime is marked by fast collapse-revival oscillations, the period of which scales as $t_{r}=\sqrt{N} / g$. In the localized regime, the tunneling is dynamically suppressed and the dimer behaves like two uncoupled Jaynes-Cummings sites. The transition region around $N_{c}^{\mathrm{qu}}$ displays multiscale oscillations. At very small photon numbers, we find two further regimes characterized by the reappearance of tunneling and secondary revivals. The richness of the quantum dynamics in the lower part of the figure is due to the finite nature of the system, namely, small $N$ and isolation from the environment.

Figure 2 displays the time-averaged quantum expectation value of the imbalance and its fluctuations as a 


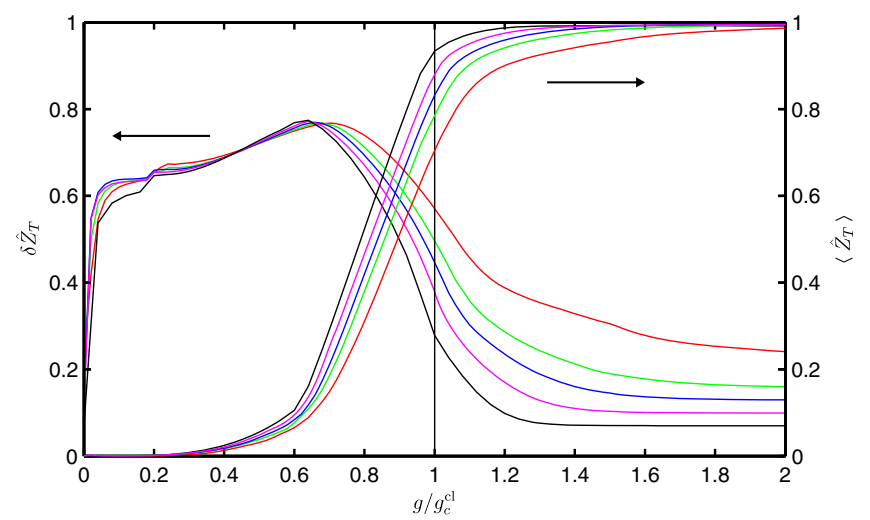

FIG. 2. Time-averaged imbalance and quantum fluctuations of the imbalance. The time-averaged imbalance for varying $g / g_{c}^{\mathrm{cl}}$, as well as its quantum fluctuations (mean squared fluctuations), for coherent states of five different mean photon numbers (red, 20; green, 40; blue, 60; magenta, 100; black, 200). Here, the total imbalance $Z_{T}$ is the difference in the number of excitations on each side (photon plus qubit excitation), normalized by the total excitations $N_{T}$ in the system. The classical prediction puts the transition at $g / g_{c}^{\mathrm{cl}}=1$. The quantum fluctuations are largest in the classically delocalized regime, and lead to a renormalization of the expected quantum value of the critical coupling $g_{c}^{\mathrm{qu}}$ downwards (i.e., requiring smaller coupling to observe localization if the total excitation number is held fixed), and hence relocating the critical photon number $N_{c}^{\text {qu }}$ upwards. This is reflected in the buildup of a finite imbalance in the region where the classical analysis predicts no net imbalance, which is, therefore, a quantum localized regime. We also observe that as the number of excitations in the system is increased, the transition gets sharper, suggesting the thermodynamic limit for this spatially finite system is given by the limit of large excitation number.

function of $g$, subject to the initialization described above. With increasing $N$, the transition becomes sharper and appears to asymptote at a $g_{c}^{\mathrm{qu}}$ that is smaller than the classical value $g_{c}^{\mathrm{cl}}$. The precise value of the renormalization of the critical coupling, $g_{c}^{\mathrm{qu}} / g_{c}^{\mathrm{cl}}$, depends on the initial quantum state. The crossover region is dominated by large quantum fluctuations and, hence, is not amenable to a simple mean-field description. A natural question to ask is, what asymptotic limit gives the semiclassical result described by a sharp transition at $g_{c}^{\mathrm{cl}}$ ? Our simulations with larger qubit spin $S$ (not shown here) indicate that the appropriate semiclassical limit is $(S, N) \rightarrow \infty$.

The above arguments apply, however, to the conservative case for which the dimer is isolated and the dynamics conserves the total excitation number, $\hat{N}_{T}=\sum_{s=L, R} \hat{\sigma}_{s}^{+} \hat{\sigma}_{s}^{-}+$ $\hat{a}_{s}^{\dagger} \hat{a}_{s}$. Below, we describe a dynamical phase transition that is of a different nature and is particular to the dimer connected to transmission lines, as studied in our experimental setup. The dynamics of such an open JaynesCummings dimer described by the master equation (3) does not conserve the total excitation number. As a consequence of this, the photon number decays exponentially, and a system initially prepared in the delocalized regime with $N_{i} \equiv N(t=0)>N_{c}^{\mathrm{qu}}$ will, at a finite time, cross the phase boundary and localize, breaking the left-right symmetry, as predicted in Refs. [22]. We note that this is distinct from the scenario described above where the transition occurs as a function of parameters $g / J$ in a system that conserves the number of excitations. This transition also differs from nonequilibrium dynamical transitions in the steady state, e.g., when a drive parameter is varied [46-50]. Interestingly, dissipation drives the system from classical behavior to quantum behavior, contrary to the standard intuition that dissipation always renders systems more classical (for previous work on a quantum to classical transition in a circuit QED realization of single-site JaynesCummings physics in the presence of an effective temperature, see Ref. [51]). The transition demonstrated in this work stands in sharp contrast to atomic and polaritonic BECs, for which the low-density dynamics is linear $[16,17,52]$, and where dissipation drives the system into a delocalized classical state [21,53].

Our experimental circuit QED realization of the JaynesCummings dimer is presented in Figs. 3(a) and 3(b). Each resonator of frequency $\nu_{c}=6.34 \mathrm{GHz}$ and linewidth $\kappa=$ $225 \mathrm{kHz}$ is individually coupled to a transmon qubit [39,40] with strength $g=190 \mathrm{MHz}$, providing a strong effective photon-photon interaction. A coupling capacitor allows photon hopping at rate $J=8.7 \mathrm{MHz}$. These parameters place the classical critical photon number at $N_{c}^{\mathrm{cl}} \approx 62$ and enable the observation of many periods of Josephson oscillations $(J \gg \kappa)$. Crucially, at fixed mean initial photon number in the localized phase, there exists an upper bound for $\kappa$ beyond which the averaged revival signal is lost, and the control afforded over dissipation in this architecture allows us to place $\kappa$ well below this bound, allowing for a good resolution of the quantum revival oscillations [23].

The device is operated in both the linear and nonlinear regimes, tuned via external flux lines $V_{L, R}$. To initialize the system [Fig. 3(c)], flux bias pulses shift both qubits far out of resonance, removing photon-photon interactions and allowing efficient population of the linear dimer modes when driven by a coherent microwave tone $V_{\text {drive }}(t)$ at frequency $\nu_{c}$ modulated by a sinusoid of frequency $J$. Once initialization is complete, and after a variable time delay $\tau$, the nonlinearity is reintroduced by flux biasing the qubits into resonance (this point is our origin of time $t=0$ ). The delay allows arranging any desired imbalance (and hence oscillation phase) at the beginning of the experiment. Here, the imbalance oscillations cover the full range $-1 \leq Z \leq+1$, unlike for BECs [20,21].

Calibrating the flux pulses requires locating bias points leading to minimal photon-photon interactions (this corresponds to the minimum of the qubit energy that gives the smallest resonator Lamb shift) for preparation, as well as resonance, where nonlinearity is largest. The low-lying spectra at these bias points is presented in Fig. 4, together 
(a)

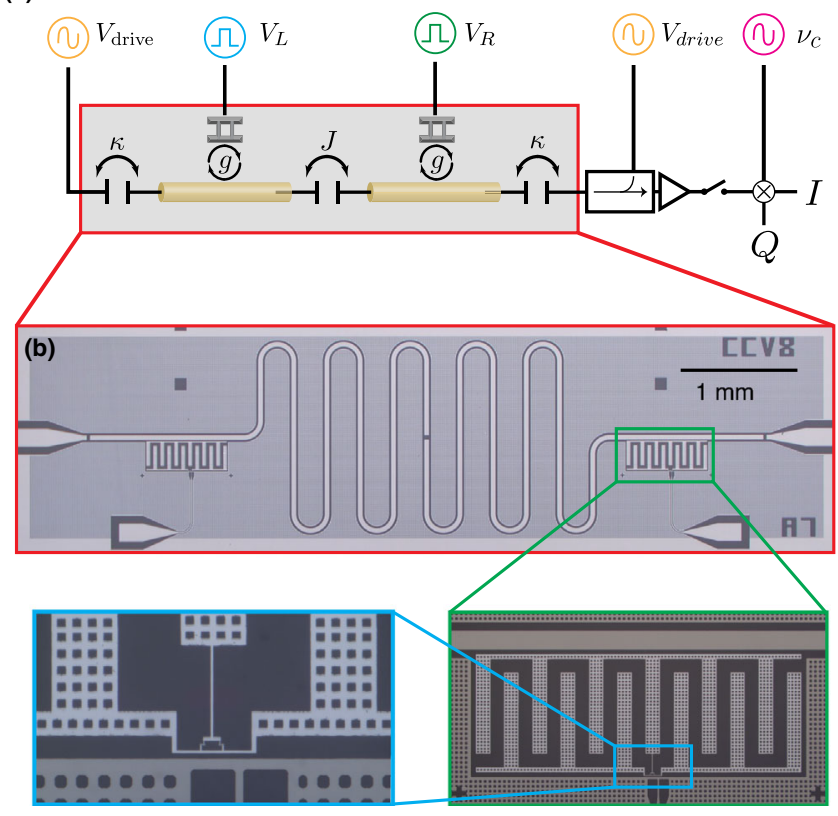

(c)

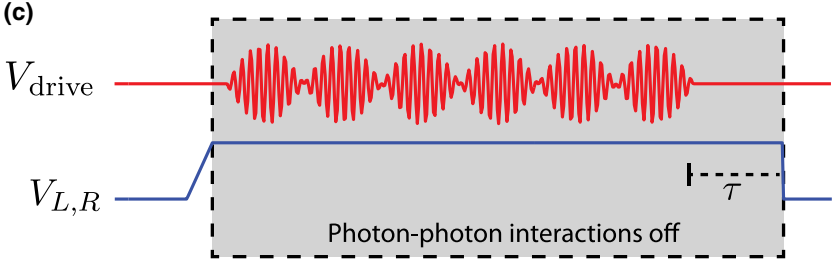

FIG. 3. Device layout and initialization routine. (a) Schematic diagram of the experiment. The Jaynes-Cummings dimer is composed of two coupled transmission-line resonators each individually coupled to a transmon qubit. Intercavity coupling $J=8.7 \mathrm{MHz}$ and cavity-qubit coupling $g=190 \mathrm{MHz}$. The resonators are driven and monitored via coupling to external transmission lines. Initialization pulse $V_{\text {drive }}$ can be applied to the left or right cavity to generate classical oscillations in the system, while fast flux pulses $V_{L, R}$ control qubit energies at nanosecond time scales. Right cavity quadratures are monitored via a homodyne measurement. When $V_{\text {drive }}$ is applied to the right cavity, a fast microwave switch is used to block the strong reflected signal. (b) Optical micrograph of the device. (c) Initialization routine pulse waveforms. Fast flux pulses $V_{L, R}$ rapidly detune both qubits to their minimum energies to turn off photon-photon interactions. While qubits are detuned, either the left or right cavity is driven with initialization pulse $V_{\text {drive }}=$ $\sin \left(2 \pi \nu_{c} t\right) \sin (2 \pi J t) \Theta(-t) \Theta(t+m / J)$, where $m$ is an integer and $\Theta$ is the Heaviside step function. Variable delay $\tau$ after the end of $V_{\text {drive }}$ allows the photon-photon interactions to be turned on at any point during the undriven linear oscillations, enabling the preparation of any desired imbalance.

with the associated single-photon nonlinearities. We develop a characterization technique useful for systems with low dissipation that relies on the Jaynes-Cummings nonlinearity, which leads to bistability with a sharp transition to a bright state as an applied continuous microwave tone is swept in power [57-59]. The threshold (a)

(b)

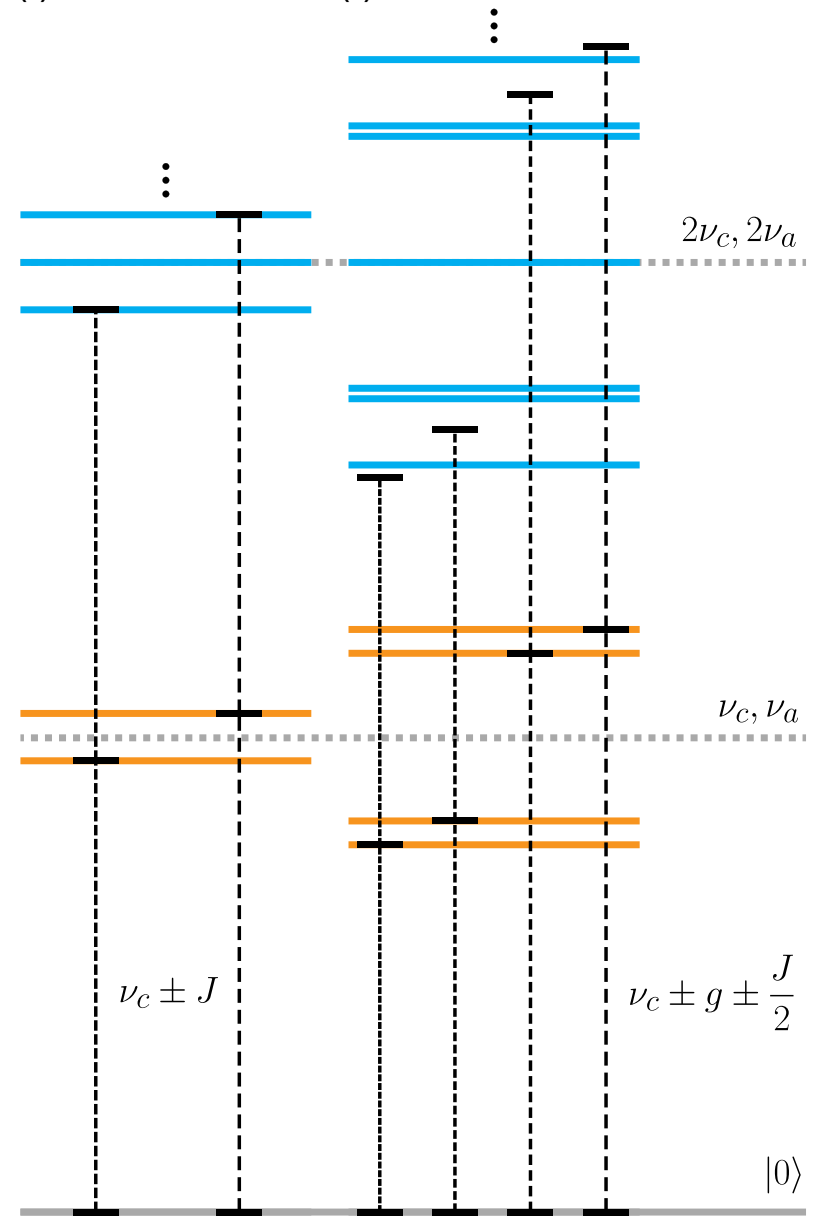

FIG. 4. Low-lying dimer spectra and nonlinearities. Spectra are shown for the two flux bias points in the experiment, without (a) and with (b) photon-photon interactions. The first (orange) and second (blue) excitation manifolds are shown along with transitions (black), where transitions from the first to second excitation manifold are the same frequency as the ground to first transitions immediately below. (a) Effective photon-photon interactions can be turned off by tuning qubits to their minimum energies. While detuned, the qubits remain in their ground states and can be ignored, resulting in a simplified spectrum of two cavities in resonance. The $J$ coupling creates two linear hybridized modes with energies $\nu_{c} \pm J$, ideal for generating full linear oscillations $[Z(t)$ oscillates between \pm 1$]$. Modulating $V_{\text {drive }}$ at $J$ generates sidebands resonant with each mode and explicitly sets the phase of the resultant linear oscillations, generating an imbalanced coherent state at $t=0$. (b) Photon-photon interactions are generated by tuning both qubits into resonance with the cavities. At this bias point, the first excitation manifold has four polariton states at energies $\nu_{c} \pm g \pm J / 2$. Strong single-photon nonlinearities are apparent, as no transitions to the second manifold match the energies of the first manifold. Because of the form of the JaynesCummings Hamiltonian, all nonlinearities are photon number dependent and lead to linear behavior at high excitation manifolds.

for this transition (above which the bright state behaves linearly) is sensitive to the frequency difference between the uncoupled mode being monitored and the 
nearest low-energy polariton mode, a useful proxy for the strength of the induced nonlinearity. Such a mapping of the two-dimensional qubit flux space identified the double minimum and resonance points (see Supplemental Material [45]).

The dynamics is observed by monitoring photons escaping one of the cavities. After amplification, the signal is mixed down with a local oscillator at $\nu_{c}$ to produce the $\hat{I}$ and $\hat{Q}$ quadratures, which are each sampled at $1 \mathrm{Gs} / \mathrm{s}$. Ensemble averaging over many trials (typically $10^{8}$ ) produces the homodyne signal and photon number (defined previously in terms of the individual quadratures).

If initialized with $N_{i}<N_{c}$ ( $N_{c}$ is taken to be the critical photon number observed in the experiment), the system localizes as soon as interactions are introduced, clearly demonstrated by strong collapse and revival of the homodyne signal $[60,61]$. These manifest as a series of lobes with peaks at integer multiples of the revival time $t_{r}=$ $\sqrt{N} / g$ and zeros at the midpoints between revivals, a uniquely quantum aspect of decoupled single-site JaynesCummings physics. Observation of clean revivals, together with an estimate of $g=190 \mathrm{MHz}$, provides a metric for the photon number in the cavity, which is then mapped back to the drive power that created the localized state. A similar calibration is done for the amplitude of the homodyne signal. Exploiting the linearity of the system during initialization, we calibrate the initial photon number throughout the full range of drive power. Figure 5(a) shows the revival signal for varying initial photon numbers below the critical value, and $\tau$ chosen to create a perfect initial imbalance $Z=-1$, placing all photons in the monitored cavity. A gap between clear Josephson oscillations (visible for $N_{i}>20$ ) and clear revival dynamics (visible for $N_{i}<13$ ) corresponds to the critical region where the revival and Josephson oscillation time scales become comparable. The inset presents a fit to the observed revival times displaying the expected $\sqrt{N}$ dependence, which is used in the calibration described above for both the initial photon number $N_{i}$ as a function of initial power and $\xi_{1}$, the homodyne signal amplitude of a coherent state with mean photon number of 1 . Figures 5(b) and 5(c) exhibit the preparation of an arbitrary imbalance, where the revival time shows a periodic variation along the vertical axis with $\tau$ (period $1 / 2 J$ ) and a relative shift between the cavities arising from the imbalance. At the values of $\tau$ corresponding to perfect imbalance $(Z= \pm 1)$, all photons are trapped in a single cavity, and the absence of any measurable signal at the other site provides strong evidence for lack of photon tunneling.

For initial photon numbers $N_{i}>N_{c}$, the system is placed in the delocalized phase. Figure 6(a) compares the dynamics without and with interactions. The former case displays quintessential linear behavior-exponential decay at a rate $\kappa=225 \mathrm{kHz}$ modulated by oscillations with frequency $2 J$, proceeding well beyond $N_{c}$ into the noise floor. Superposed is a typical example of the nonlinear dissipation-driven classical to quantum transition, initially displaying Josephson oscillations enveloped by an exponential decay. The slightly faster effective decay rate $\left(\kappa^{\prime}=265 \mathrm{kHz}\right)$ can be attributed to small qubit dissipation and dephasing (as verified by simulations), which play a more significant role with the qubits in resonance and satisfies the condition for strong single-photon nonlinearity $g \gg \kappa^{\prime}$, a regime not accessible to current exciton-polariton (a)

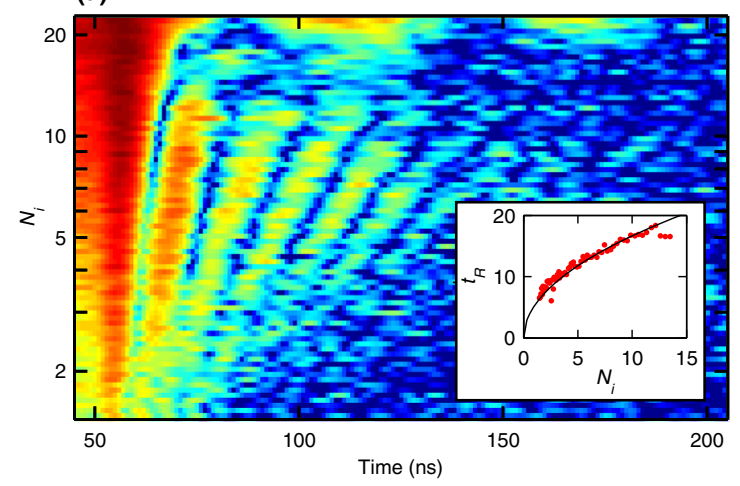

(b)

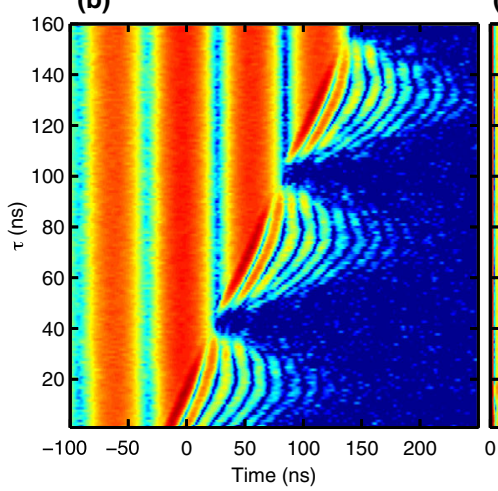

(c)

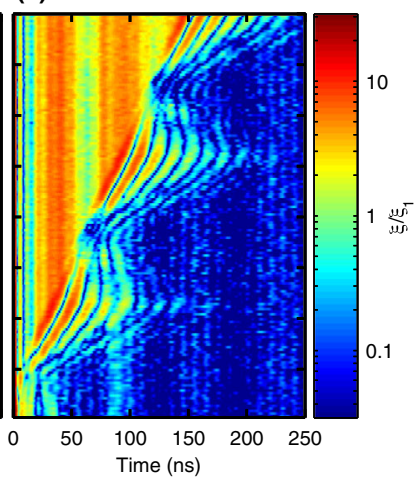

FIG. 5. Self-trapped regime. (a) Homodyne signal dynamics of the right cavity as a function of initial photon number and time. A 345ns $(3 / J)$ initialization pulse $V_{\text {drive }}$ ending at $t=0$ generates linear oscillations between the left and right cavities while photon-photon interactions are off. The oscillations proceed for a time $\tau$ such that a perfect imbalance $(Z=-1)$ is established when interactions are turned on. For $N_{i}<N_{c}$, the system is localized and the right cavity displays fast collapse and revival oscillations while the left cavity (not shown) remains empty. Inset: Fitting revival time $t_{r}$ as a function of drive power to the expected $\sqrt{N}$ dependence allows drive power to be mapped to initial photon number (at $t=0$ ), as well as the calibration of $\xi_{1}$, the homodyne signal of a coherent state with mean occupation of one photon. (b),(c) Revival time variation as a function of $\tau$ for $V_{\text {drive }}$ applied to the left (left-hand panel) and right (righthand panel). As $\tau$ is varied, the full range of imbalance is observed. Here, the same initialization scheme as in (a) is used with $N_{i}=8$, which produces the cleanest collapse and revival signature. When driving the right cavity, strong reflections during $V_{\text {drive }}$ give rise to signal distortion during the dynamics (visible in the right-hand panel) that are mitigated by using a microwave switch. 

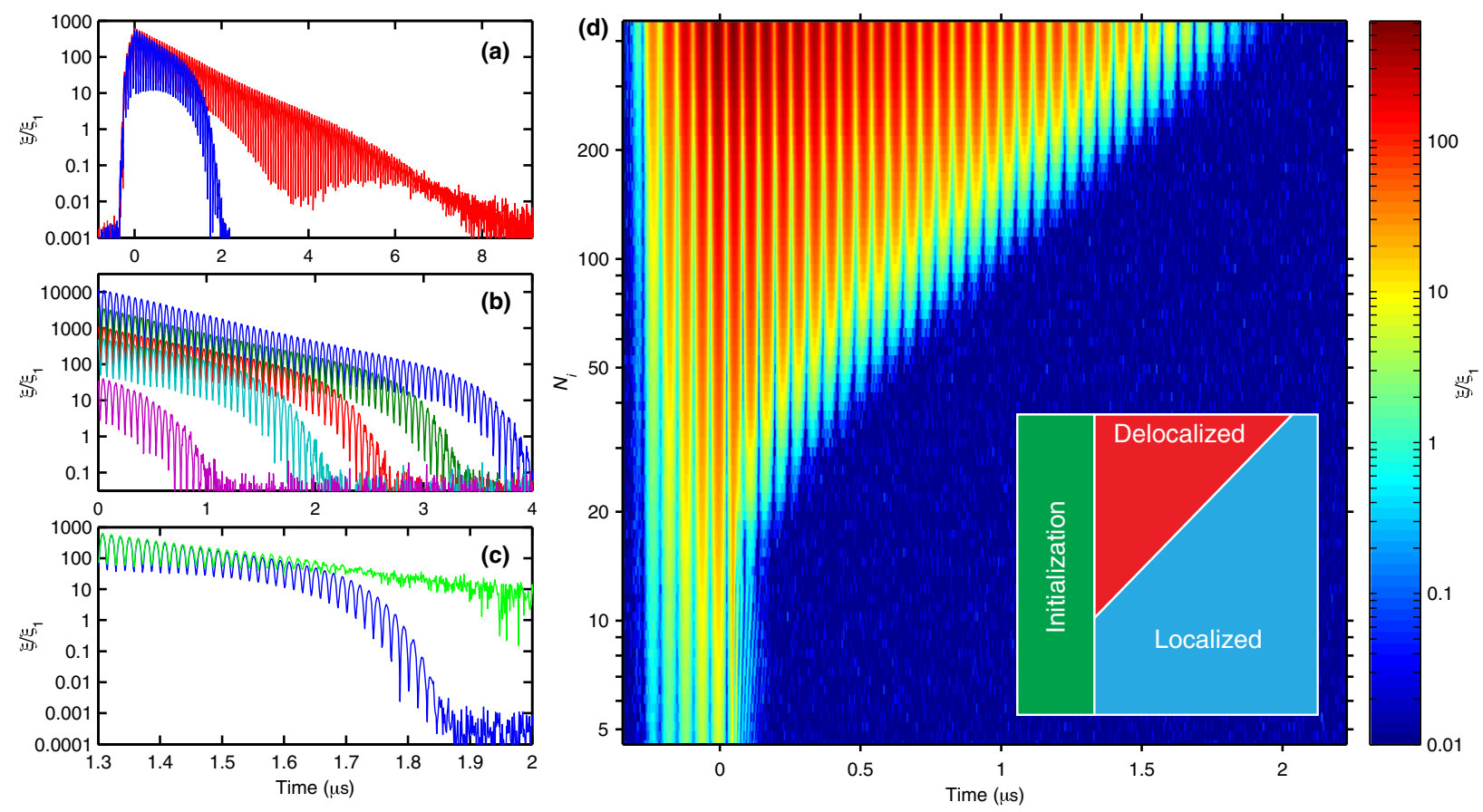

FIG. 6. Dissipation-driven transition and phase diagram. (a) Comparison of homodyne signal when photon-photon interactions are off (red) and on (blue), with $N_{i}>N_{c}$. The same initialization pulse $V_{\text {drive }}$ was used as in Fig. 5. With interactions off, the system undergoes oscillations and exponential decay at rate $\kappa$, which can be observed for several microseconds. Significantly, the presence of interactions causes superexponential decay of the homodyne signal as $N$ approaches $N_{c}$, a signature of crossover into the localized regime. (b) The time of onset of superexponential decay $t_{c}$ shifts with initial photon number, as shown here for initialization pulses with varying drive power lasting $11.5 \mu \mathrm{s}(100 / J)$. The use of a long initialization pulse makes it possible to drive the system to very large initial photon numbers (top to bottom: $N_{i} \approx 12000,3800,1100,550,40$ ), but introduces complications (see Supplemental Material for more details [45]). (c) Directly measuring the photon number (green) reveals that incoherent photons remain in the system after the homodyne signal (blue) has undergone superexponential decay. Oscillations in the photon number can also be observed to die out, as critical slowingdown constrains the envelope of oscillations, finally leaving only exponential decay. Here, $V_{\text {drive }}$ is $1.15 \mu \mathrm{s}(10 / J)$ and $\tau=1 \mu \mathrm{s}$. Background voltages leading to distortion of the signal were removed from the photon number measurement. (d) Reconstructing the phase diagram by monitoring the homodyne signal as a function of initial photon number and time. At high powers, the dynamical transition from linear oscillations to localized behavior is marked by superexponential decay, while at low powers the collapse and revival signatures of localized behavior are observed. A $345 \mathrm{~ns}(3 / J)$, an initialization pulse $V_{\text {drive }}$ ending at $t=0$ is used with $\tau=65 \mathrm{~ns}$, corresponding to an initial imbalance $Z \approx-0.6$. Inset: Illustration of the phase diagram showing the different dynmical regimes.

BECs [21]. The exponential decay of the oscillations later gives way to a superexponential drop in homodyne signal, a signature of the crossover from delocalized to localized behavior: photon escape is a stochastic process, and for a given trial the photon number falls below $N_{c}$ at a random time, with an average time dependent on the initial photon number. When approaching this point, the Josephson oscillations become nonlinear, exhibiting a critical slowing-down [22]. Oscillations of different trials within an ensemble dephase with respect to each other, and individual trials once localized exhibit very rapid collapse; thus, ensemble averages of $\hat{I}$ and $\hat{Q}$ die out faster than exponentially, and only trials where $N(t) \gg N_{c}$ continue to contribute to the homodyne signal. Figure 6(b) shows the observed homodyne dynamics for various initial photon numbers, revealing the logarithmic dependence of the critical time to reach the transition on the initial photon number, $t_{c} \sim \frac{1}{\kappa^{\prime}} \log \left(N_{i} / N_{c}\right)$.
Unlike the homodyne signal, photon number measurement is insensitive to the coherence of field in the monitored cavity. Additionally, the dispersion between individual trials arising from critical slowing-down does not cause the photon number to decay superexponentially. Figure 6(c) compares homodyne measurement to photon number for the same initial condition. For short times, the two signals match, demonstrating a high degree of coherence within the ensemble. In contrast to the superexponential decay of the homodyne signal, we see an exponential decay of the photon number.

The homodyne observation maps out a dynamical phase diagram as a function of initial photon number $N_{i}$ and time, as displayed in Fig. 6(d). An applied drive during an initialization phase where photon-photon interactions are off initiates Josephson oscillations. An undriven and noninteracting region lasting a constant time $\tau$ follows, continuing the oscillations, at the end of which the 


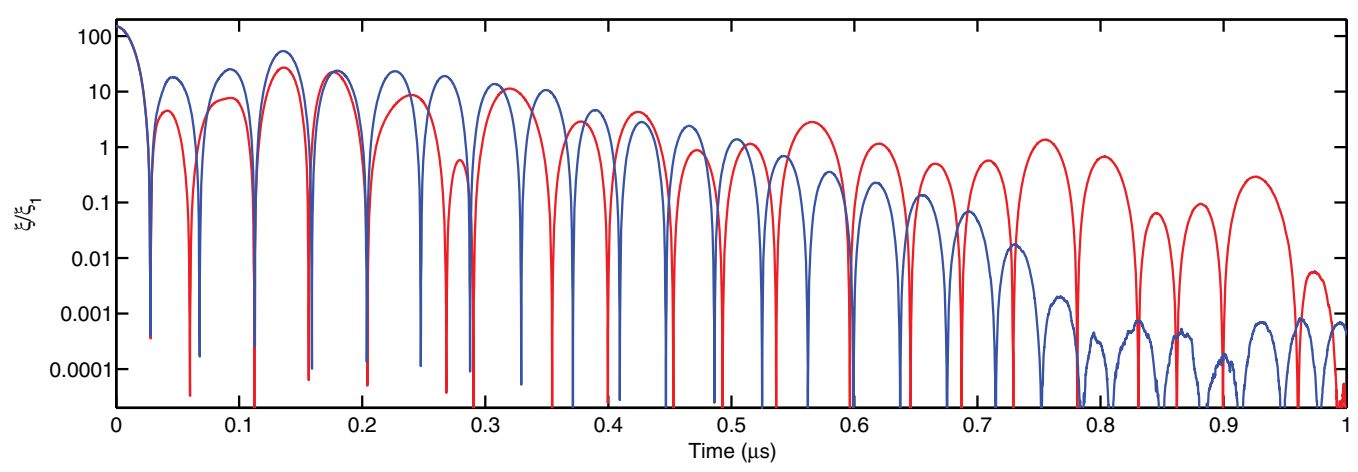

FIG. 7. Simulated homodyne signal without and with dissipation. A comparison of the homodyne dynamics (shown on a logarithmic scale, normalized to $\xi_{1}$, the homodyne signal of a single-photon coherent state) without dissipation (red curve) and with (blue curve, averaged over 1000 walks in a quantum trajectory simulation of the master equation). The initial state is a coherent one with a mean of 150 photons on the left, no photons on the right, and the qubits in the ground state. The unitary dynamics (no dissipation) exhibits nonlinear oscillations due to the competition between intercavity tunneling and qubit coupling, in the delocalized regime not very far above $N_{c}^{\mathrm{qu}}$. As the tunneling is less sensitive to dissipative effects, the tendency is for dissipation to linearize the oscillations. The early overshoot in the homodyne signal results from transient behavior in the initial dynamics of the system.

nonlinearity is rapidly switched on. For $N_{i}>N_{c}$, there exists a delocalized, interacting phase extending for a time scaling logarithmically with $N_{i}$, before crossing over into a localized regime. These oscillations are the dominant feature visible for initial photon numbers greater than 20. With an initialization such that $N_{i}<N_{c}$, the system immediately localizes as interactions are switched on, maintaining the trial coherence and, hence, visibility of the collapse and quantum revival in the homodyne signal. These revivals can be seen as the very short time scale features occurring below $N_{i}=13$.

We find that the experimentally observed critical photon number $\left(13 \lesssim N_{c} \lesssim 20\right)$ is at variance with the classical prediction $\left(N_{c}^{\mathrm{cl}} \approx 62\right)$ and, moreover, with the full closed quantum dynamics simulation (placing $N_{c}^{\mathrm{qu}} \approx 120$, as seen in Fig. 1). Another feature of the experimental data is the regularity of oscillations close to the transition for $N_{i} \approx N_{c}$, which stands in contrast to the irregular oscillations in the full quantum dynamics shown in Fig. 1. To understand whether dissipation is behind these observations, we also perform quantum trajectory simulations of the open dimer [that effectively solves the master equation (3)]. Figure 7 compares quantum trajectory simulations of an initially coherent state at photon number above $N_{c}^{\mathrm{qu}}$, without and with dissipation. The presence of dissipation is found to linearize the intercavity oscillations leading to a homodyne signal that exhibits regular harmonic oscillations, as observed in the experiments. The variance of the observed critical number from the predicted value might arise from the role of dissipation in the dynamical transition, fluctuations in the initial state due to imperfections in preparation, the effect of the higher transmon levels that may be important in the early time dynamics (though the detuning of the higher levels should suppress their contributions), and the dynamics associated with quenching in the vicinity of a critical point.
We demonstrate a nonequilibrium localization transition in a strongly correlated open photonic system. Our deliberate choice of parameters places the transition in a region at the margin of accessibility to classical simulation of the quantum dynamics, providing a test of the reliability of this architecture as a platform for quantum simulation of open systems [62]. We expect that future experiments will build on this work to explore the intricate dynamical behavior of lattices when dissipation plays a fundamental role, and which lie beyond what classical simulators can replicate.

The authors would like to acknowledge fruitful discussions with Devin Underwood, Will Shanks, Srikanth Srinivasan, David Huse, Marco Schiró, Hassan Shapourian, Stephan Mandt, and Steve Girvin. The Princeton work was supported by The Eric and Wendy Schmidt Transformative Technology Fund, the U.S. National Science Foundation through the Princeton Center for Complex Materials (DMR-0819860) and CAREER awards (Grants No. DMR-0953475 and No. DMR-1151810), the David and Lucile Packard Foundation, and U.S. Army Research Office Grant No. W911NF-11-1-0086. S.S. acknowledges support through an Ambizione grant of the Swiss National Science Foundation. J.R. and D. S. contributed equally to this work.

[1] H. M. Jaeger and A. J. Liu, Condensed-Matter and Materials Physics: The Science of the World Around Us (National Academies Press, Washington DC, 2007).

[2] W. H. Zurek, Decoherence, Einselection, and the Quantum Origins of the Classical, Rev. Mod. Phys. 75, 715 (2003).

[3] A. Polkovnikov, K. Sengupta, A. Silva, and M. Vengalattore, Colloquium: Nonequilibrium Dynamics of 
Closed Interacting Quantum Systems, Rev. Mod. Phys. 83, 863 (2011).

[4] M. Rigol, V. Dunjko, and M. Olshanii, Thermalization and Its Mechanism for Generic Isolated Quantum Systems, Nature (London) 452, 854 (2008).

[5] S. Trotzky, Y.-A. Chen, A. Flesch, I. P. McCulloch, U. Schollwöck, J. Eisert, and I. Bloch, Probing the Relaxation towards Equilibrium in an Isolated Strongly Correlated One-Dimensional Bose Gas, Nat. Phys. 8, 325 (2012).

[6] T. Langen, R. Geiger, M. Kuhnert, B. Rauer, and J. Schmiedmayer, Local Emergence of Thermal Correlations in an Isolated Quantum Many-Body System, Nat. Phys. 9, 640 (2013).

[7] M. Lewenstein, A. Sanpera, and V. Ahufinger, Ultracold Atoms in Optical Lattices: Simulating Quantum Many-Body Systems (Oxford University Press, Oxford, England, 2012).

[8] A. Blais, R.-S. Huang, A. Wallraff, S. M. Girvin, and R. J. Schoelkopf, Cavity Quantum Electrodynamics for Superconducting Electrical Circuits: An Architecture for Quantum Computation, Phys. Rev. A 69, 062320 (2004).

[9] A. Wallraff, D. I. Schuster, A. Blais, L. Frunzio, R.-S. Huang, J. Majer, S. Kumar, S. M. Girvin, and R. J. Schoelkopf, Strong Coupling of a Single Photon to a Superconducting Qubit Using Circuit Quantum Electrodynamics, Nature (London) 431, 162 (2004).

[10] B. D. Josephson, Possible New Effects in Superconductive Tunnelling, Phys. Lett. 1, 251 (1962).

[11] K. K. Likharev, Superconducting Weak Links, Rev. Mod. Phys. 51, 101 (1979).

[12] K. Sukhatme, Y. Mukharsky, T. Chui, and D. Pearson, Observation of the Ideal Josephson Effect in Superfluid ${ }^{4} \mathrm{He}$, Nature (London) 411, 280 (2001).

[13] J. Javanainen, Oscillatory Exchange of Atoms between Traps Containing Bose Condensates, Phys. Rev. Lett. 57, 3164 (1986).

[14] S. V. Pereverzev, A. Loshak, S. Backhaus, J. C. Davis, and R. E. Packard, Quantum Ocillations between Two Weakly Coupled Reservoirs of Superfluid ${ }^{3} \mathrm{He}$, Nature (London) 388, 449 (1997).

[15] S. Backhaus, S. Pereverzev, R. W. Simmonds, A. Loshak, J. C. Davis, and R. E. Packard, Discovery of a Metastable $\pi$ State in a Superfluid ${ }^{3} \mathrm{He}$ Weak Link, Nature (London) 392, 687 (1998).

[16] A. Smerzi, S. Fantoni, S. Giovanazzi, and S. R. Shenoy, Quantum Coherent Atomic Tunneling between Two Trapped Bose-Einstein Condensates, Phys. Rev. Lett. 79, 4950 (1997).

[17] S. Raghavan, A. Smerzi, S. Fantoni, and S. R. Shenoy, Coherent Oscillations between Two Weakly Coupled BoseEinstein Condensates: Josephson Effects, $\pi$ Oscillations, and Macroscopic Quantum Self-Trapping, Phys. Rev. A 59, 620 (1999).

[18] S. Levy, E. Lahoud, I. Shomroni, and J. Steinhauer, The a.c. and d.c. Josephson Effects in a Bose-Einstein Condensate, Nature (London) 449, 579 (2007).

[19] K. G. Lagoudakis, B. Pietka, M. Wouters, R. André, and B. Deveaud-Plédran, Coherent Oscillations in an ExcitonPolariton Josephson Junction, Phys. Rev. Lett. 105, 120403 (2010).

[20] M. Albiez, R. Gati, J. Fölling, S. Hunsmann, M. Cristiani, and M. K. Oberthaler, Direct Observation of Tunneling and
Nonlinear Self-Trapping in a Single Bosonic Josephson Junction, Phys. Rev. Lett. 95, 010402 (2005).

[21] M. Abbarchi, A. Amo, V. G. Sala, D. D. Solnyshkov, H. Flayac, L. Ferrier, I. Sagnes, E. Galopin, A. Lemaître, G. Malpuech, and J. Bloch, Macroscopic Quantum SelfTrapping and Josephson Oscillations of Exciton Polaritons, Nat. Phys. 9, 275 (2013).

[22] S. Schmidt, D. Gerace, A. A. Houck, G. Blatter, and H. E. Türeci, Nonequilibrium Delocalization-Localization Transition of Photons in Circuit Quantum Electrodynamics, Phys. Rev. B 82, 100507 (2010).

[23] S. Haroche and J. M. Raimond, Exploring the Quantum: Atoms, Cavities, and Photons (Oxford University Press, Oxford, England, 2006).

[24] R. P. Feynman, Simulating Physics with Computers, Int. J. Theor. Phys. 21, 467 (1982).

[25] S. Lloyd, Universal Quantum Simulators, Science 273, 1073 (1996).

[26] A. A. Houck, H. E. Türeci, and J. Koch, On-Chip Quantum Simulation with Superconducting Circuits, Nat. Phys. 8, 292 (2012).

[27] S. Schmidt and J. Koch, Circuit QED Lattices: Towards Quantum Simulation with Superconducting Circuits, Ann. Phys. (Amsterdam) 525, 395 (2013).

[28] A. D. Greentree, C. Tahan, J. H. Cole, and L. C. L. Hollenberg, Quantum Phase Transitions of Light, Nat. Phys. 2, 856 (2006).

[29] M. J. Hartmann, F. G. S. L. Brandão, and M. B. Plenio, Strongly Interacting Polaritons in Coupled Arrays of Cavities, Nat. Phys. 2, 849 (2006).

[30] D. G. Angelakis, M. F. Santos, and S. Bose, Photon-BlockadeInduced Mott Transitions and xy Spin Models in Coupled Cavity Arrays, Phys. Rev. A 76, 031805 (2007).

[31] J. Koch and K. Le Hur, Superfluid-Mott-Insulator Transition of Light in the Jaynes-Cummings Lattice, Phys. Rev. A 80, 023811 (2009).

[32] M. Leib and M. J. Hartmann, Bose-Hubbard Dynamics of Polaritons in a Chain of Circuit Quantum Electrodynamics Cavities, New J. Phys. 12, 093031 (2010).

[33] F. Nissen, S. Schmidt, M. Biondi, G. Blatter, H. E. Türeci, and J. Keeling, Nonequilibrium Dynamics of Coupled Qubit-Cavity Arrays, Phys. Rev. Lett. 108, 233603 (2012).

[34] A variant of the previously referenced circuit-QED-based architectures using networks of nonlinear resonators is presented in Ref. [35].

[35] M. Leib, F. Deppe, A. Marx, R. Gross, and M. J. Hartmann, Networks of Nonlinear Superconducting Transmission Line Resonators, New J. Phys. 14, 075024 (2012).

[36] J. Koch, A. A. Houck, K. L. Hur, and S. M. Girvin, TimeReversal-Symmetry Breaking in Circuit-QED-Based Photon Lattices, Phys. Rev. A 82, 043811 (2010).

[37] D. I. Tsomokos, S. Ashhab, and F. Nori, Using Superconducting Qubit Circuits to Engineer Exotic Lattice Systems, Phys. Rev. A 82, 052311 (2010).

[38] D. L. Underwood, W. E. Shanks, J. Koch, and A. A. Houck, Low-Disorder Microwave Cavity Lattices for Quantum Simulation with Photons, Phys. Rev. A 86, 023837 (2012).

[39] J. Koch, T. M. Yu, J. Gambetta, A. A. Houck, D. I. Schuster, J. Majer, A. Blais, M. H. Devoret, S. M. Girvin, and 
R. J. Schoelkopf, Charge-Insensitive Qubit Design Derived from the Cooper Pair Box, Phys. Rev. A 76, 042319 (2007).

[40] J. A. Schreier, A. A. Houck, J. Koch, D. I. Schuster, B. R. Johnson, J. M. Chow, J. M. Gambetta, J. Majer, L. Frunzio, M. H. Devoret, S. M. Girvin, and R. J. Schoelkopf, Suppressing Charge Noise Decoherence in Superconducting Charge Qubits, Phys. Rev. B 77, 180502 (2008).

[41] The square-root dependence of the critical coupling (6) on the photon number is a consequence of the JaynesCummings nonlinearity [42]. At small photon numbers, the Jaynes-Cummings ladder is strongly nonlinear, favoring localization.

[42] J. M. Fink, M. Göppl, M. Baur, R. Bianchetti, P. J. Leek, A. Blais, and A. Wallraff, Climbing the Jaynes-Cummings Ladder and Observing Its Nonlinearity in a Cavity QED System, Nature (London) 454, 315 (2008).

[43] J. Gea-Banacloche, Collapse and Revival of the State Vector in the Jaynes-Cummings Model: An Example of State Preparation by a Quantum Apparatus, Phys. Rev. Lett. 65, 3385 (1990).

[44] J. Gea-Banacloche, Atom-and Field-State Evolution in the Jaynes-Cummings Model for Large Initial Fields, Phys. Rev. A 44, 5913 (1991).

[45] See Supplemental Material at http://link.aps.org/ supplemental/10.1103/PhysRevX.4.031043 for details of the characterization of the Jaynes-Cummings dimer allowing us to locate the two qubit flux bias points; present a toy model capturing the essential physics of the superexponential decay; give more detail on the driven dynamics during the initialization phase; and comment on the difficulty of simulating the open system quantum dynamics of our class of devices.

[46] F. Brennecke, R. Mottl, K. Baumann, R. Landig, T. Donner, and T. Esslinger, Real-Time Observation of Fluctuations at the Driven-Dissipative Dicke Phase Transition, Proc. Natl. Acad. Sci. U.S.A. 110, 11763 (2013).

[47] B. Öztop, M. Bordyuh, Ö. E. Müstecaploğlu, and H. E. Türeci, Excitations of Optically Driven Atomic Condensate in a Cavity: Theory of Photodetection Measurements, New J. Phys. 14, 085011 (2012).

[48] M. Kulkarni, B. Öztop, and H. E. Türeci, CavityMediated Near-Critical Dissipative Dynamics of a Driven Condensate, Phys. Rev. Lett. 111, 220408 (2013).

[49] L. M. Sieberer, S. D. Huber, E. Altman, and S. Diehl, Dynamical Critical Phenomena in Driven-Dissipative Systems, Phys. Rev. Lett. 110, 195301 (2013).
[50] P. H. Chavanis, F. Baldovin, and E. Orlandini, NoiseInduced Dynamical Phase Transitions in Long-Range Systems, Phys. Rev. E 83, 040101 (2011).

[51] J. M. Fink, L. Steffen, P. Studer, Lev S. Bishop, M. Baur, R. Bianchetti, D. Bozyigit, C. Lang, S. Filipp, P. J. Leek, and A. Wallraff, Quantum-to-Classical Transition in Cavity Quantum Electrodynamics, Phys. Rev. Lett. 105, 163601 (2010).

[52] B. Juliá-Díaz, D. Dagnino, M. Lewenstein, J. Martorell, and A. Polls, Macroscopic Self-Trapping in Bose-Einstein Condensates: Analysis of a Dynamical Quantum Phase Transition, Phys. Rev. A 81, 023615 (2010).

[53] Other types of interactions of BECs with an environment can lead to localization. As an example, measurementinduced localization arising from spontaneous emission of photons from cold atoms and its implications for optical lattices have been studied in Refs. [54-56].

[54] M. Holland, S. Marksteiner, P. Marte, and P. Zoller, Measurement Induced Localization from Spontaneous Decay, Phys. Rev. Lett. 76, 3683 (1996).

[55] F. Gerbier and Y. Castin, Heating Rates for an Atom in a FarDetuned Optical Lattice, Phys. Rev. A 82, 013615 (2010).

[56] H. Pichler, A. J. Daley, and P. Zoller, Nonequilibrium Dynamics of Bosonic Atoms in Optical Lattices: Decoherence of Many-Body States due to Spontaneous Emission, Phys. Rev. A 82, 063605 (2010).

[57] M. Boissonneault, J. M. Gambetta, and A. Blais, Improved Superconducting Qubit Readout by Qubit-Induced Nonlinearities, Phys. Rev. Lett. 105, 100504 (2010).

[58] L. S. Bishop, E. Ginossar, and S. M. Girvin, Response of the Strongly Driven Jaynes-Cummings Oscillator, Phys. Rev. Lett. 105, 100505 (2010).

[59] M. D. Reed, L. DiCarlo, B. R. Johnson, L. Sun, D. I. Schuster, L. Frunzio, and R. J. Schoelkopf, High-Fidelity Readout in Circuit Quantum Electrodynamics Using the Jaynes-Cummings Nonlinearity, Phys. Rev. Lett. 105, 173601 (2010).

[60] G. Rempe, H. Walther, and N. Klein, Observation of Quantum Collapse and Revival in a One-Atom Maser, Phys. Rev. Lett. 58, 353 (1987).

[61] M. Greiner, O. Mandel, T. W. Hänsch, and I. Bloch, Collapse and Revival of the Matter Wave Field of a Bose-Einstein Condensate, Nature (London) 419, 51 (2002).

[62] P. Hauke, F. M. Cucchietti, L. Tagliacozzo, I. Deutsch, and M. Lewenstein, Can One Trust Quantum Simulators?, Rep. Prog. Phys. 75, 082401 (2012). 